\title{
Enhanced Angular Topographic Backscatter Electron Filtering
}

\author{
D.W. Phifer ${ }^{1}$, J. Pecher ${ }^{2}$, P. Valasek ${ }^{2}$, M. Petrek ${ }^{2}$ and T. Vystavel ${ }^{2}$ \\ 1. FEI Company B.V., Eindhoven, The Netherlands. \\ 2. FEI Brno, Brno, Czech Republic.
}

Angular segmentation of detected signal in a SEM can be used to segment topographic and compositional contrast as has been shown with concentric ring solid state detection [1]. Subdividing the topographic contrast further can yield additional information on the direction of sample surfaces and allow clear interpretation of topography. Signal intensity is influenced by both sample topography and composition and the ability to separate one from the other may be useful in estimating scale of topography.

Solid state detector diodes have been introduced with the ability to address concentric segment rings around the beam exit to separate high angle and low angle electrons (as shown in Figure 1). This works quite well to distinguish between composition and density on the inner rings closest to the beam and topographic information which is more prominent on the outer rings [1]. What is perhaps missing is the relative direction of the surface as the shading is uniform from one side of the image to the other. Conventional segmentation of the detector such that there are 4 "quadrant" segments does not allow removal of atomic number effects on the topographic information as the inner (composition) and outer (topographic) information are both within a given signal collection area. Since BSE distribution is dependent on both composition and topography, the effect of a high atomic number region with topography may be exaggerated in respect to an adjacent low atomic number region due to the combined signal brightness and contrast. This direct result is used for calculating height of topography based on contrast and brightness differences. The inability to separate the brightness due to composition could skew results if brightness and contrast are used as the determining factor for height, even when this is compared to a reference "off-axis" or $5^{\text {th }}$ segment signal and this could be a contributing factor for why this data is not so accurate [2].

Subdividing the outer "topographic" rings of concentric ring detector segments into three 60 degree "arch" segments, eliminates the composition information and allows characterization of directional information from "pure" topographic content. An example of this is shown in Figure 2, where images from the inner ring and the three segments are shown. Shadowing is based on the direction of the sample surface.

Directional signal segmentation is an emerging way to get new sample surface information. Angular filtering has the potential to create more accurate surface height representations in SEM imaging where composition can be removed from the equation or conversely used to refine the signal interpretation to yield better estimations because of the compositional signal differences. New ability to separate signal provides valuable insight into topographic information and presents new opportunity to use different, direction-rich data for surface mapping and height measurements.

\section{References:}

[1] T. Vystavel, XIVth International Conference on Electron Microscopy (2011).

[2] A. Agrawal et al, European Conference on Computer Vision (ECCV) (2006) p21. 

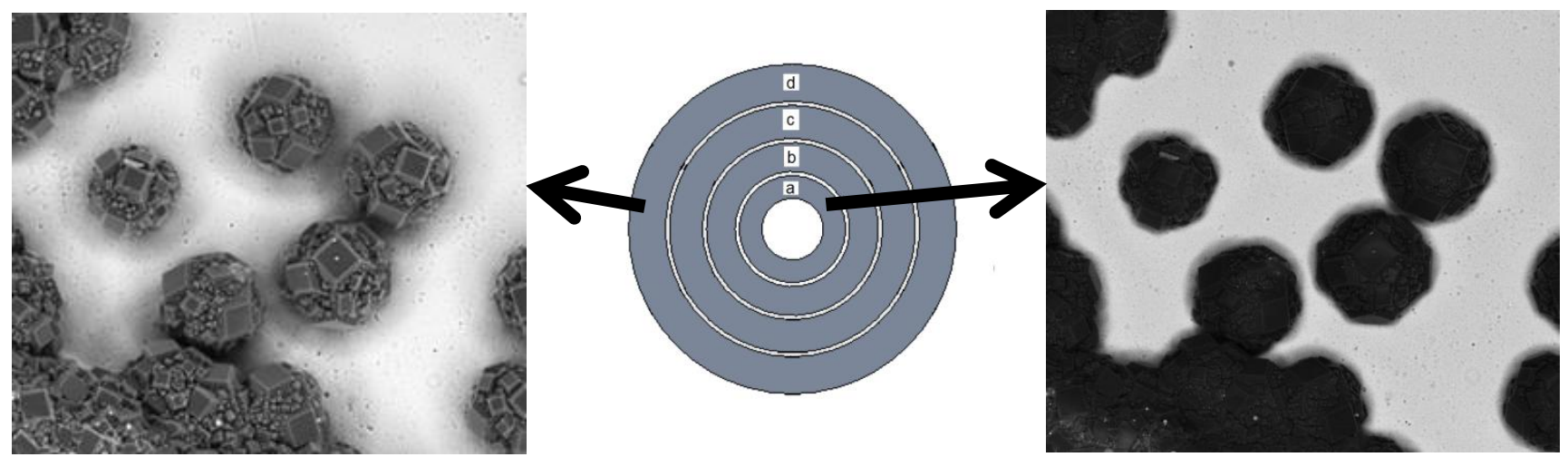

Figure 1. Angular filtering with concentric ring segmentation clearly separates the atomic (right image) and topographic (left image) content from BSE signal. Field width is $\sim 23$ microns.

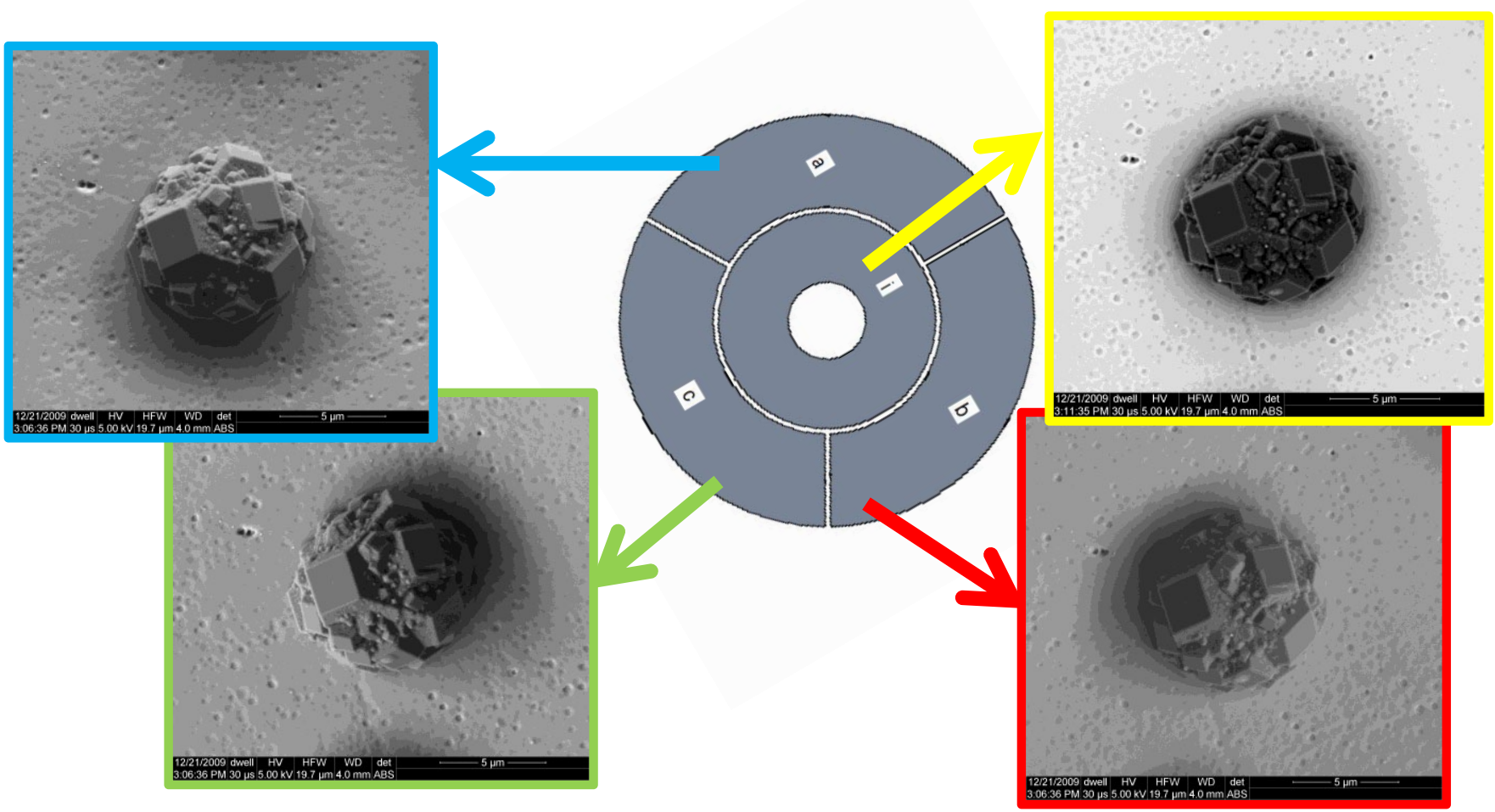

Figure 2. With an angular segmentation of the outer detector segments, separation of the topographic component is possible with angular direction yielding perspective shadows. This new information can be used to interpret the direction of slope in an image area in relation to neighboring pixels. 